\title{
Effect of methane on stability of plasma in a MW-assisted hydrogen-methane plasma
}

\author{
Swaminathan Prasanna, A. Michau, C. Rond, S. Farhat, K. Hassouni, A. Gicquel \\ LSPM, CNRS 3407, Universite Paris 13 Villetaneuse France
}

MW assisted Hydrogen-methane plasmas have been used extensively for growth of carbon materials such as CVD diamond, graphene and carbon nano-tubes. Graphene is usually grown at low pressures ( $>25$ mbar) where as CVD diamond can be grown at relatively high pressures. Microwave radiation energizes the few free electrons to very high temperature, which collide with heavy species resulting in a self-sustaining plasma. In a conventional resonating MW cavity, the plasma occurs close to one of the available anti-nodes in the reactor and the substrate is placed closed to this anti-node enabling growth of diamond or graphene. The quality of the deposit depends on the coupling between microwave and the plasma which again depends on various factors such as MW power, pressure, concentration of the precursor gases etc. Understanding the coupling between the microwave and the plasma is critical for design and optimization of efficient MW reactors.

In this article, we discuss the effects of concentration of methane in a hydrogen plasma for carbon material processing in a MW assisted bell jar reactor which has been used for growing CVD diamonds and graphene at LSPM [1] (schematic shown in Fig. 1). The reactor is characterized by having two anti-nodes, one close to the substrate and the other close to the top of the bell jar reactor. At normal operating conditions, the plasma is formed close to the substrate which is desirable. However as the MW power is increased, the plasma can become unstable as two plasma zones can be formed.

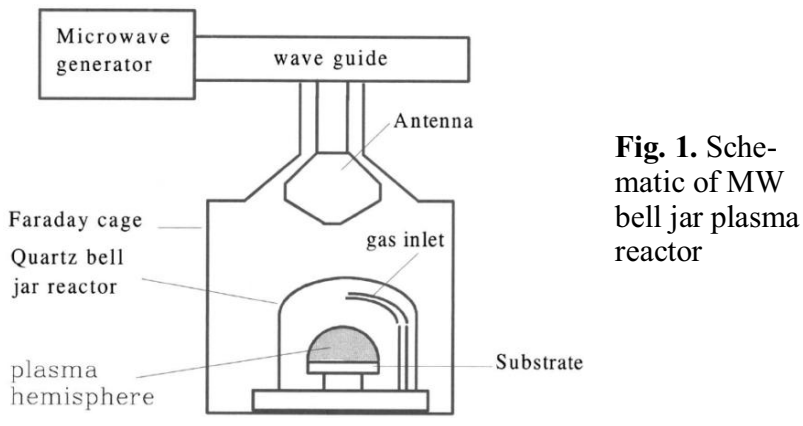

We have observed that the addition of methane into the reactor affects the general plasma characteristics. The threshold MW power density (PMWD) at which the plasma becomes unstable have been measured for various concentration of methane in a MW bell jar reactor. This is achieved by visualizing the plasma emissive value at different conditions. Fig 2. shows the effect of concentration of methane on the threshold microwave power density. Addition of methane lowers the threshold microwave power density. Self-consistent simulations [2] have also been performed for these conditions ( 25 mbar). The difference between the numerical simulations and experiments may be due to uncertainty of absorbed microwave power, and also sensitivity of the numerical scheme. It has to be noted that only input power is available and there is no quantitative measurement of the MW power absorbed by the plasma. However, similar trends have been observed with experiments and numerical simulations as seen in Fig. 2.

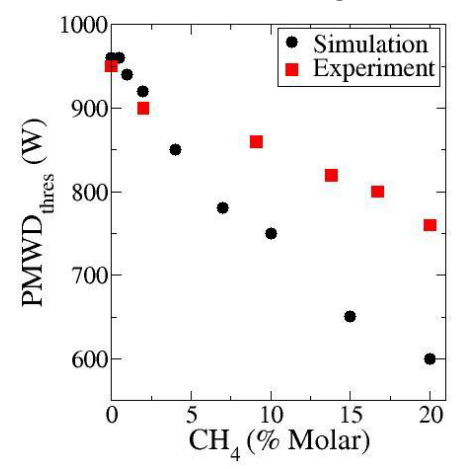

Fig. 2. Threshold Microwave power density at which plasma becomes unstable in a MW bell jar plasma reactor at 25 mbar

Similar results have been observed numerically for different reactor pressures. The present results are important because the effective range of operation of the MW reactor depends on the precursor gas concentration and pressure. The present observation can be explained by the dominant ions present in the reactor. In a pure hydrogen plasmas, the dominant ions under these conditions is $\mathrm{H}_{3}{ }^{+}$. However, with the addition of methane, the dominant ion species shifts to $\mathrm{CH}_{5}{ }^{+}$and $\mathrm{C}_{2} \mathrm{H}_{3}{ }^{+}$depending on the concentration of methane. As the hydrocarbon ions are heavier compared to the hydrogen ions, the diffusion of hydrocarbon ions is much lower than the hydrogen ions. Consequently, higher ion density and thus electron density (due to electro-neutrality and ambi-polar diffusion prevailing under these conditions) is observed in the plasma core. This means the hydrocarbon plasmas observe higher power dissipation close to the substrate than the hydrogen plasmas. This has been confirmed from the numerical simulations. As a result the size of the plasma for with hydrocarbons is larger than the pure hydrogen plasmas for same MW power conditions Formation of secondary plasmas occurs when sufficient electrons are available at the second anti-node. This process is accelerated for a hydrocarbon plasma due to large size of plasma at the same input microwave power.

The present results show that the MW-plasma coupling is affected by the concentration of methane. To design efficient and optimum reactors, it will be important to understand the effects of the precursor gases on MWplasma coupling. Further studies would be conducted to understand the MW-plasma coupling in different gas discharges at wide range of operating conditions.

Acknowledgements. This work was supported by ANR through two projects ANR-11-LABX-086/ ANR11-IDEX-0005-02 and ANR2011BS09029.

\section{References}

1. Gicquel, A., Hassouni, K., Farhat, S., Breton, Y., Scott, C.D., Lefebvre, M. and Pealat, M. Spectroscopic analysis and chemical kinetics modeling of a diamond deposition plasma reactor. // Diamond and Related Materials, 1994, 3, 581-586.

2. Prasanna, S., Rond, C., Michau, A., Hassouni, K. and Gicquel, $A$. Effect of buoyancy on power deposition in microwave cavity hydrogen plasma source.// Plasma Sources Science and Technology, 2016, 25,045017 